\title{
Marcos temáticos y multifuncionalidades de los huertos comunitarios en el área urbana de A Coruña (Galicia): un estudio de caso
}

\author{
Thematic frameworks and multifunctionalities of the community gardens in the \\ urban area of A Coruña (Galicia): A case study \\ JUAN SORIANO-BAEZA \\ Universidade da Coruña \\ ORCID: 0000-0003-3756-1736 \\ jusorbae@gmail.com \\ RAIMUNDO OTERO-ENRÍQUEZ \\ Universidade da Coruña, Grupo de Estudios Territoriales \\ ORCID: 0000-0002-8074-3765 \\ raimundo.otero@udc.es \\ ESTEFANÍA CALO \\ Universidade da Coruña, Grupo de Estudios Territoriales \\ ORCID: 0000-0003-3846-5869 \\ estefania.calo@udc.es
}

\section{RESUMEN}

Desde finales del siglo XX, la agricultura urbana y periurbana desarrollada en huertos comunitarios ha pasado de ser una actividad residual e informal, a convertirse en un objeto de estudio que ocupa un papel emergente en las políticas de planificación de las áreas urbanas. El primer objetivo de este artículo es el de acotar teóricamente el concepto de huerto urbano comunitario, y realizar una recopilación de la literatura actual sobre una realidad cada vez más común en las tramas metropolitanas. El segundo objetivo se centra en exponer los resultados de una investigación sociológica, de tipo cuantitativo, realizada en tres huertos comunitarios de la periferia de la ciudad de A Coruña (Galicia). De este modo, al hilo de la revisión teórica previa, se podrán extrapolar al contexto territorial gallego, de modo inédito, datos y reflexiones globales sobre la multifuncionalidad y las motivaciones que sostienen la producción de esta particular tipología de agricultura periurbana.

Palabras clave: Huertos periurbanos comunitarios, huertos urbanos comunitarios, agricultura urbana, motivaciones, Galicia, A Coruña. 


\begin{abstract}
Since the late 20th century, urban and peri-urban agriculture developed in community gardens has evolved from being a residual and informal activity, to that of an object of study that has an emerging role in the planning policies of urban areas. The first objective of this article is to delimit theoretically the concept of urban community garden, and compile current literature about a reality increasingly common in metropolitan plots.

The second objective is to present the results of a sociological research with quantitative methodology carried out in three community gardens in the city of A Coruña (Galicia). Together with the previous theoretical revision, global data and reflections on multifunctionality and the motivations that support this particular typology of peri-urban agriculture can be extrapolated in an unprecedented way to the Galician territorial context.
\end{abstract}

Keywords: Peri-urban community gardens, urban community gardens, urban agriculture, motivations, Galicia, A Coruña.

\title{
1. INTRODUCCIÓN
}

A partir de la Primera Revolución Industrial, el estudio académico de la relación entre el campo y la ciudad se ha entendido a partir del poder centrípeto y expansivo de la ciudad, que se ha ido imponiendo funcionalmente al espacio circundante ocupado por actividades agrarias y ganaderas. Partiendo de esta óptica, se puede empezar a explicar la escasa atención que se la ha prestado, durante gran parte del siglo XX y desde las ciencias del territorio, a la pervivencia de complejas formas de agricultura entreveradas en las tramas metropolitanas centrales y periféricas.

Es un lugar común en la creciente literatura que en las últimas décadas ha tratado de estudiar el fenómeno de la agricultura urbana y periurbana (en adelante AUP), recordarnos de partida que los/as residentes en áreas metropolitanas supondrán en el año 2050 el $66,4 \%$ de la población mundial ${ }^{1}$. Por tanto, resulta totalmente caduco seguir manteniendo una relación antagónica entre la agricultura y los espacios urbanos por dos motivos: primero, porque dicho antagonismo no ha existido en tanto en cuanto la AUP es tan vieja como el nacimiento de las ciudades (Verdaguer, 2013); segundo, porque la promoción de esta actividad se ha revelado en los últimos años como un instrumento de primera importancia para el mantenimiento de la seguridad alimentaria, el ahorro energético y la reducción global de emisiones contaminantes a la atmósfera en cualquier país (Clinton et al., 2018).

El interés creciente por la AUP se localiza, especialmente, en países con índices modestos de desarrollo humano (Mougeot, 2005, 2011). Sin embargo, en las últimas décadas del siglo XX, se empieza a poner el foco en otros contextos territoriales como

1 Véanse los datos recogidos en las últimas World Urbanization Prospects, the 2014 revision, en http://esa. un.org/unpd/wup/index.htm (enlace consultado el 19/06/2018). 
el norteamericano y europeo ${ }^{2}$. En ellos, la tipología en la que se manifiesta la AUP es muy diversa, y tiene una gran variedad de actores implicados, de escalas productivas y de posibles localizaciones en la red metropolitana. Siendo más concretos, la AUP se puede bifurcar en dos grandes tipologías: la primera, circunscrita a los huertos urbanos individuales o comunitarios de pequeña autoproducción; la segunda, sujeta a los principios del mercado y de gran escala productiva (Duží et al., 2017: 131).

Hecha esta breve contextualización y aclaración conceptual, y tras haber presentado el marco temático de referencia, se presentará una investigación centrada en describir la idiosincrasia de una forma concreta de AUP: la de tres huertos comunitarios de autoproducción situados en escenarios diferentes del área periurbana de A Coruña (Galicia).

\section{HUERTOS COMUNITARIOS URBANOS Y PERIURBANOS: DEFINICIÓN Y PRINCIPALES LÍNEAS TEMÁTICAS DE ANÁLISIS}

La definición amplia de huerto urbano urbano y periurbano (en adelante HUPC) lleva implícita la discusión de los conceptos de "comunidad" (miembros que solo comparten una determinada proximidad geográfica, afinidad o institución), de "producción" (autoconsumo, venta o donación), o de "propiedad y gestión" (pública, asociativa o privada) (Pudup, 2008). De igual modo, las características de los huertos son muy variables atendiendo a una localización urbana, con una alta densidad poblacional y edificatoria, $\mathrm{u}$ otra de tipo periurbano o exurbano con disponibilidades de suelo superiores.

Aproximarnos a una definición precisa de lo que es un HUPC, exige que esta sea, en esencia, amplia. Podemos decir, por tanto, que un huerto comunitario es un espacio gestionado por un colectivo de personas que comparten alguna característica común, y donde se cultivan alimentos. Esta descripción se localiza en varias contribuciones académicas (Guitart et al., 2012) y resulta útil en tanto en cuanto ofrece una característica diferencial con otros tipos de AUP desarrollados en terrazas, parcelas o jardines privados desconectados de esta connotación de "grupo" o "comunidad" (Soriano, 2017: 16).

A partir de la década de los setenta, bajo un contexto de crisis energética y económica, en Estados Unidos aparecen en toda su geografía - especialmente en Nueva Yorkhuertos urbanos de tipo comunitario, desligados de una actividad agrícola de subsistencia, que son concebidos como espacios colectivos de regeneración metropolitana y expresión de múltiples movimientos ecologistas y contraculturales (Taylor, 2010; Morán y Aja, 2011). Desde este punto de inflexión, la extensión de los HUPC, especialmente en países "desarrollados" y "en vías de desarrollo", ha sido extraordinaria ; de hecho, la FAO en los últimos años asume como perentoria la necesidad de incorporar estos espacios tanto a la

\footnotetext{
2 Un ejemplo claro de este tipo de trabajos es el de Morán (2009) sobre los huertos urbanos en tres ciudades europeas (Londres, Berlín y Madrid).

3 Véase, de estos países, una selección de estudios de caso en Soriano (2017: 14-16).
} 
agenda de la planificación de las áreas urbanas, como a programas alimenticios de ámbito nacional (Integrated Food Security Support Service, 2011).

Guitart et al. (2012: 366), a través de una búsqueda sistemática de literatura escrita en inglés sobre huertos comunitarios (community gardens), muestra como las investigaciones se suceden desde varias disciplinas y se publican en diferentes revistas especializadas que, por orden de importancia, son: geografía, medio ambiente y planeamiento, sociedad y cultura, salud, educación, economía y biología. También, en la misma aportación de Guitart et al. (2012: 367), se observa como una gran parte de los artículos analizados hacen referencia a la diversidad cultural y de estatus económico de los/as usuarios/as de los HUPC, y a una mayor abundancia de estudios llevados a cabo en zonas de baja renta per cápita.

Soriano (2017: 20) propone la siguiente clasificación temática, atendiendo a las investigaciones realizadas a partir de 1990, fundamentalmente en Estados Unidos ${ }^{4}$ :

- Salud (física y mental) y producción de alimentos frescos y de alta calidad nutritiva.

- Impactos generados de tipo económico, laboral y educacional.

- Nuevos usos del espacio urbano.

- Nuevas formas de interacción social, ocio y expresión cultural.

- El empoderamiento de organizaciones colectivas y de movilización social.

En el contexto español, la producción científica sobre los HUPC está experimentando en los últimos años un aumento parejo a la creciente eclosión de esta actividad agraria en diversas ciudades españolas. En este sentido, resulta muy valioso el estudio de Ballesteros (2016) que contabiliza en el año 2015, a partir de un rastreo de noticias publicadas en medios digitales, un total de casi 500 HUPC en todo el país, siendo una mayoría los de titularidad pública, y siendo la Comunidad Autónoma andaluza la que acoge un mayor número de parcelas (más de un 20\% del total). En el año 2000, este autor identificaba solo 15, dato que contrastado con el anterior, evidencia la rápida extensión de estos espacios de cultivo en España 5 .

Merece una mención el estudio de corte cualitativo realizado por Michelini y Méndez (2017), en el que se contabilizan un total de 60 HUPC en el conjunto de la Comunidad de Madrid $^{6}$. Ambos autores vinculan la emergencia de este tipo de AUP al cuestionamiento de un modelo de urbanización neoliberal, y la recuperación de una agricultura de proximidad; no obstante, precisan, su práctica es abordada por una gran variedad de colectivos (desde "clases sociales creativas" hasta movimientos sociales antisistema).

4 Véase una completa gama de trabajos citados para cada una de estas líneas de investigación, en Soriano (2017: 16-20).

5 Para el mismo año, Ballesteros (2016) estima que la superficie ocupada por el total de HUPC es superior a los dos millones de $\mathrm{m}^{2}$; en el año 2000, la superficie ocupada no llegaba a los $150.000 \mathrm{~m}^{2}$.

6 En la ciudad de Madrid también resulta de especial interés el estudio de caso propositivo de Simón (2017) sobre el barrio de Bellas Vistas. Asimismo, hay que destacar el análisis de los huertos de Sevilla realizado por Puente (2012). 
Precisamente, las indagaciones sobre el perfil de usuario/a de los HUPC en España son variopintas. Simón (2016: 37) describe un/a cultivador/a de clase media, de alto nivel formativo y una ocupación laboral estable7. En cambio, en un ámbito "micro" como el de los huertos de Tarrasa, según Domene y Saurí (2016: 289) el perfil mayoritario es el de una persona jubilada, de clase trabajadora, que realiza esta actividad por una importante variedad de razones (metas personales, apoyo familiar, recuperación de un vínculo con el mundo rural, etc.).

Por último, son destacables los trabajos que dan cuenta de la faceta multifuncional de los HUPC. En concreto, seleccionamos las aportaciones, entre otras posibles, de Cabo et al. (2014) sobre los aspectos motivacionales (relacionados con la salud, la interacción social y el ocio) que impulsan a un colectivo de jubilados/as en Valladolid a cultivar un huerto urbano. También, dentro de la línea temática del empoderamiento y de la movilización social, señalar las reflexiones de Camps-Calvet et al. (2014) sobre los huertos barceloneses como expresión, en tiempos de crisis económica, de una forma de resistencia a un estilo de vida urbano hegemónico y de corte neoliberal.

\section{EL CONTEXTO GALLEGO: DESCRIPCIÓN DEL CAMPO, OBJETIVOS Y METODOLOGÍA}

Con la excepción de las aportaciones de Bisquert i Pérez $(2013,2017)$ sobre las implicaciones socioeducativas asociadas a los huertos urbanos de Santiago de Compostela, no han sido localizadas a día de hoy otras contribuciones académicas en Galicia relacionadas con algunas de las líneas temáticas identificadas en el epígrafe precedente ${ }^{8}$. Tampoco se dispone de una información mínimamente sistematizada sobre el número y la distribución territorial de los HUPC gallegos; por tanto, esta ha sido una de las primeras lagunas analíticas a cubrir.

Siguiendo el mismo proceder que Ballesteros (2016), se han rastreado noticias publicadas en medios digitales y, especialmente, en webs institucionales de los ayuntamientos que integran las grandes áreas urbanas o metropolitanas gallegas (en adelante AU) ${ }^{9}$. En el año 2018, siguiendo estos criterios, se han localizado un total de 69 HUPC; según datos de Ballesteros (2016), en el año 2015 se localizaban en Galicia 20 espacios con

7 Los datos de este estudio incluyen además de huertos urbanos gestionados colectivamente, a cooperativas y grupos de consumo autogestionados.

8 Sí que existen, no obstante, diferentes iniciativas asociativas destinadas a dar visibilidad y promover este fenómeno emergente en las áreas urbanas gallegas. Destacamos, en este sentido, a la Asociación Galega de Horticultura Urbana (véase http://hortaurbana.blogspot.com -enlace consultado el 19/06/2018-).

9 Para fijar el ámbito de búsqueda municipal urbano y periurbano de Galicia, resulta óptima la unidad espacial de gran área urbana, propuesta por el Ministerio de Fomento (véase metodología en https:// www.fomento.gob.es/mfom/lang_castellano/_especiales/siu/atlas/ -enlace consultado el 19/06/2018-). En total son cinco las grandes áreas urbanas gallegas, nucleadas por siete ciudades y otros 25 municipios periurbanos (véase Figura 1). 
estas características. En definitiva, al igual que en el resto de España, estamos hablando de una actividad de rápida expansión y cada vez más presente en el entramado urbano y periurbano gallego.

El perfil "tipo" de huerto tiene una extensión de $2.500 \mathrm{~m}^{2}$, y está estructurado por unas 40 parcelas de alrededor de $50 \mathrm{~m}^{2}$ (por supuesto, este conjunto inicial de datos tiene que ser asumido con cautela ${ }^{10}$. Atendiendo a su distribución, el mayor número de HUPC se concentra en las AU de Vigo-Pontevedra (36,8\% del total), de A Coruña (20,6\% del total) y de Santiago de Compostela (27,9\%) (véase Figura 1).

El 79,4\% de estos espacios agrarios son de titularidad pública-municipal; el 8,8\% son gestionados directamente por diversas asociaciones o fundaciones; el porcentaje restante corresponde, en su mayor parte, a iniciativas privadas. Estas cifras entroncan con la importancia que el "municipalismo", ante la falta de políticas autonómicas, estatales y comunitarias de referencia ${ }^{11}$, ha tenido a la hora de elaborar un abanico de ordenanzas o normativas reguladoras de los HUPC, que los alejan de los clichés de la irregularidad e irrelevancia administrativa. Por tanto, sin querer desmerecer la importancia de otras iniciativas asociativas y privadas, son los principales ayuntamientos -especialmente el coruñés y santiagués durante el último lustro- los que están apuntalando y organizando, a través de gobernanzas participativas ${ }^{12}$ y pioneros marcos reguladores ${ }^{13}$, la práctica comunitaria de la agricultura urbana en Galicia.

Circunscribiéndonos a partir de ahora al caso de los huertos periurbanos del área metropolitana de A Coruña, se plantea una investigación de tipo exploratorio y descriptivo, cuyas metas se materializan en los siguientes objetivos:

- Llevar a cabo un análisis de la segmentación demográfica, ocupacional y económica de la población que participa en huertos comunitarios de diferente titularidad.

- Analizar la relación entre la edad y la situación económica de los/as cultivadores/ as de los huertos, y las principales motivaciones que subyacen a la práctica de la AUP.

- Ilustrar, progresivamente, las incógnitas y particularidades nacidas de este estudio de caso exploratorio que precisarían futuras investigaciones.

10 De hecho, para el cálculo de la superficie y número de parcelas de los HUPC, de un total de 69 casos, solo se han tenido datos fiables de 17.

11 Precisar que, una vez finalizados los trabajos en 2016 de la ambiciosa red de investigación Urban Agriculture Europe (ligada al programa COST), parece que en el futuro mediato la AUP será un elemento importante a incluir, entre otros programas comunitarios (McEldowney, 2017), en próximas reformas de la Política Agraria Común (véase http://www.cost.eu/media/cost_stories/urban-agriculture-europe-suntapped-potential -enlace consultado el 19/06/2018-).

12 En el trabajo de Soriano (2017) se describe el proceso participativo de diseño y organización de las Ecohortas del Concello de A Coruña (véase también http://ecohortas.coruna.gal/\#inicio -enlace consultado el 19/06/2018-).

13 Sobre esta cuestión, léase el Libro Blanco das hortas urbanas municipais de Santiago de Compostela (véase https://compostelaverde.santiagodecompostela.gal/info/-enlace consultado el 19/06/2018-). 


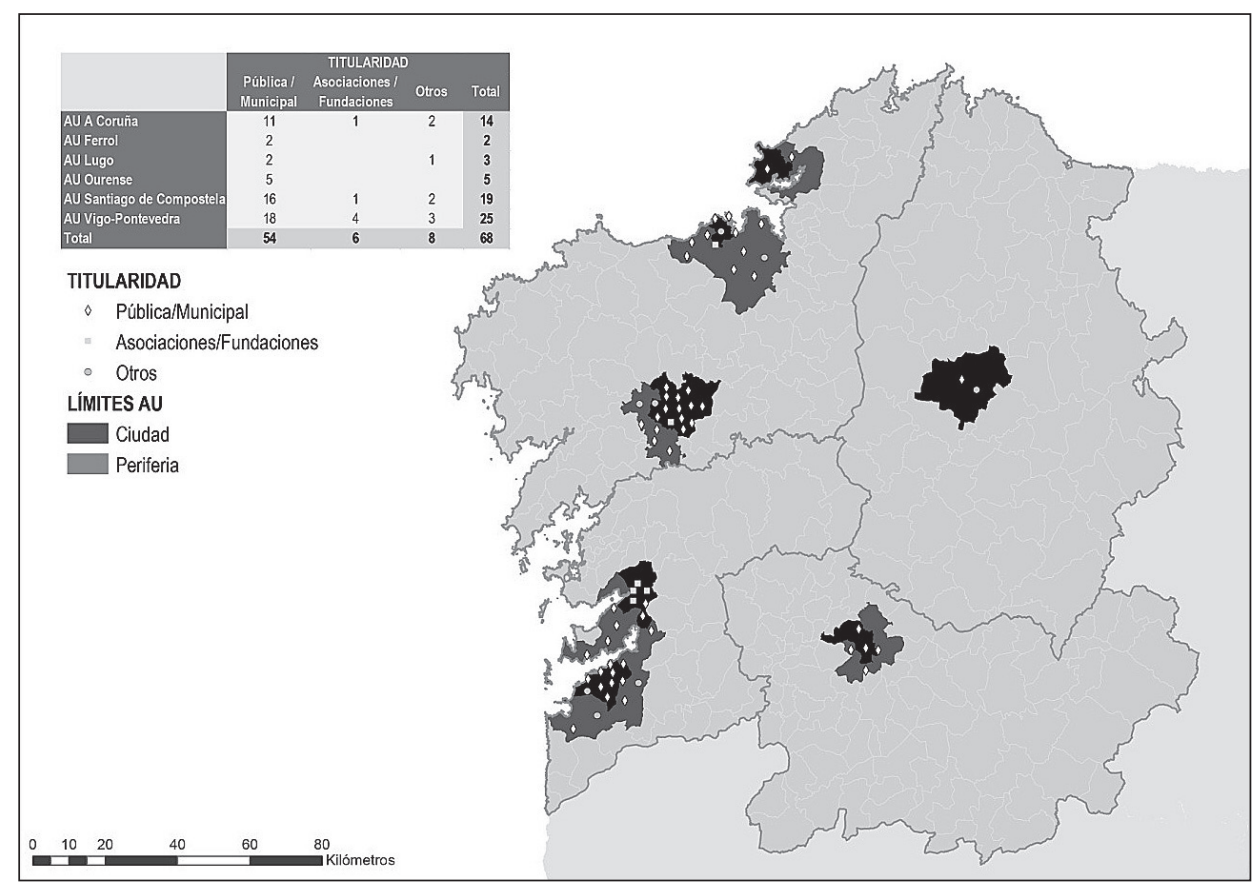

Figura 1. Localización de los HUPC en Galicia según gran área urbana (AU) y titularidad.

Fuente: elaboración propia.

Para alcanzar estos tres objetivos se ha recurrido a una metodología cuantitativa, decisión que, en primer término, sienta unas primeras coordenadas, contrastadas estadísticamente, sobre un fenómeno hasta ahora muy poco analizado en Galicia; en segundo lugar, se distancia del predominio de investigaciones cualitativas referidas a los HUPC (Guitart et al., 2012).

El soporte del enfoque metodológico es la realización de una encuesta a los/as cultivadores/as de tres huertos comunitarios de la órbita periurbana de la ciudad de A Coruña. Dos de ellos se sitúan en el municipio metropolitano de Culleredo, de titularidad municipal; el primero inicia su actividad en el año 2010 en el jardín del Centro Cultural Villa Melania; el segundo, se pone en funcionamiento dos años después en el Jardín Botánico de O Burgo. El tercero se sitúa en el barrio periférico de Feáns (municipio de A Coruña), siendo gestionado por una asociación independiente que inicia su andadura también en el 2010 (véase Figura 2). 

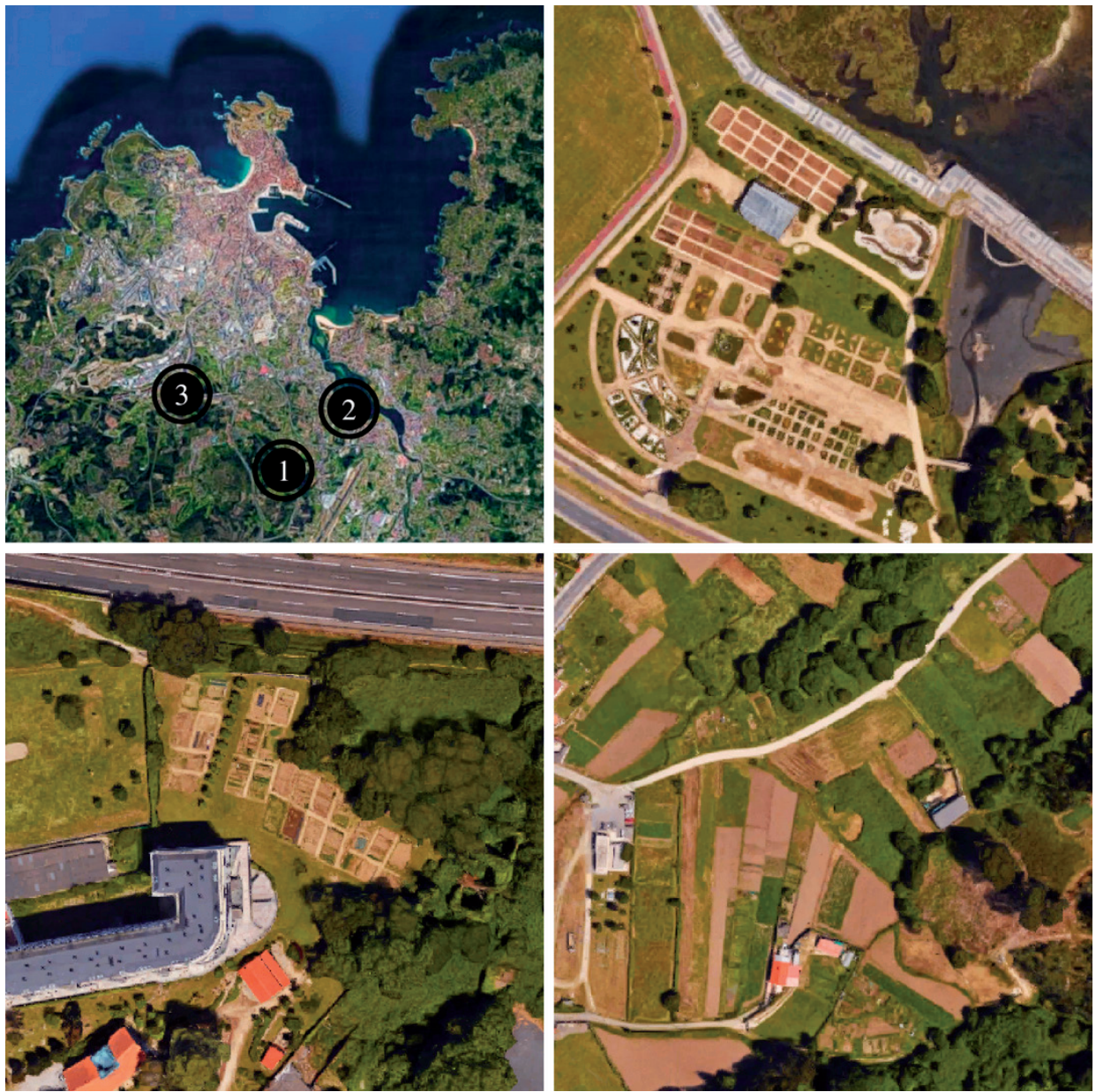

Figura 2. Localización y fotografías aéreas de los huertos estudiados.

NOTA. De izquierda a derecha y de arriba abajo: Localización de los huertos; (1) huerto municipal de CulleredoO Burgo; (2) huerto municipal de Culleredo-Villa Melania; (3) huerto de Feáns.

Fuente: Google Earth.

En los huertos comunitarios situados en Culleredo el/la titular de cada parcela ha de estar empadronado/a en dicho municipio; en el caso del huerto de Feáns, no existe más requisito que inscribirse en la asociación y que esta disponga de un espacio libre. El parcelario de los tres huertos es gestionado independientemente por el conjunto de usuarios/ as. Los dos huertos en Culleredo suman un total de 55 parcelas de unos $50 \mathrm{~m}^{2}$ cada una, con su correspondiente titular; en los huertos de Feáns se pueden encontrar diferentes tamaños de parcela, pero la media también ronda $\operatorname{los} 50 \mathrm{~m}^{2}$, para un total de 130 socios/as ${ }^{14}$.

14 En este huerto, además, se gestionan colectivamente $500 \mathrm{~m}^{2}$ destinados a cultivos que demandan mayores extensiones de terreno -como patatas o nabos-. 
El destino de la producción en los tres espacios está orientado de modo exclusivo al autoconsumo. En el caso de Culleredo la venta de los productos obtenidos queda expresamente prohibida ${ }^{15}$, y así queda recogido a la vez en los estatutos y reglamento interno de la asociación que gestiona el huerto de Feáns. Señalada esta última característica común, estos espacios son seleccionados porque se amoldan íntegramente al concepto y características de los HUPC ya expuestas a partir de la contribución de Pudup (2008).

Debido a la imposibilidad de acceder a la población objetivo fuera de los tres huertos, la estrategia de recogida de información consistió en intentar encuestar, de modo aleatorio, al máximo número de cultivadores/as, y acercarse todo lo posible al total del universo (185 hortelanos/as). Durante los meses de junio de 2016 a mayo de 2017, se realizaron más de veinte visitas al campo, recogiéndose un total de 86 encuestas (un 46,5\% de la población objeto de estudio) ${ }^{16}: 47$ (un 54,7\% de la muestra) se realizaron en los huertos municipales de Culleredo; 39 (el 45,3\% restante) en el huerto de Feáns.

\section{ANÁLISIS DE RESULTADOS}

\subsection{Perfil de los/as cultivadores/as y práctica cotidiana}

Trazar un "perfil-tipo" de cultivador/a supone el primer objetivo analítico a alcanzar. Para ello, se enumeran los siguientes atributos sociodemográficos del conjunto de encuestados/as (véase Tabla I):

- Hay una mayor proporción de hombres que de mujeres $(60,5 \%$ y $39,5 \%$ respectivamente). El grupo de edad de 18 a 34 años es minoritario (7\%) y los dos colectivos restantes, de 35 a 54 años y de más de 55 años, tienen la misma presencia (46,5\%). La media de edad se sitúa en 52,1 años, cifra similar a la manejada en otras investigaciones (Alaimo et al., 2008).

- La mayor presencia de hombres se mantiene en los tres grupos de edad estudiados, aspecto que no es usual en trabajos en los que se analiza el género predominante en los/as hortelanos/as de los HUPC (Glover et al., 2005).

- En cuanto al nivel de estudios máximo alcanzado, destaca el grupo con graduado escolar/ESO o EGB (36\%). El porcentaje de personas con formación universitaria es también relativamente alto $(29,1 \%)$, seguido por el colectivo con formación profesional de grado medio o superior $(20,9 \%)$.

- Atendiendo a la situación laboral, el 44,2\% de los/as entrevistados/as son trabajadores/as. No obstante, es destacable la proporción de personas en situación

15 Consúltese la ordenanza reguladora de los huertos en el BOP n ${ }^{\circ}$ 122, miércoles 30 de Junio de 2010 (véase http://bop.dicoruna.es/bopportal/ -enlace consultado el 19/06/2018-).

16 Trasladando esta cifra a un modelo probabilístico, se ha obtenido por tanto una muestra aleatoria simple con un error absoluto del 7,7\%, dado un nivel de confianza del $95 \%$, asumiendo que $\mathrm{p}=\mathrm{q}=0,5$, y tras aplicar la pertinente corrección (puesto que $\mathrm{f}=\mathrm{n}^{\prime} / \mathrm{N}>0,05$ ). 
de inactividad, o bien por causa del desempleo (23,3\% del total), o bien por ser jubiladas y/o perceptoras de una pensión (23,3\%). Estas dos últimas casuísticas son comunes a las detectadas en los análisis de Ballesteros (2016).

- Con respecto a la auto-valoración de la situación económica del hogar del/de la cultivador/a, casi la mitad de los/as entrevistados/as se sitúa en una posición "intermedia" (47,7\%); el grupo con una valoración "baja" supone un 27,9\% del total, y el grupo en mejor situación el 24,4\%.

Tabla I. Caracterización de los perfiles de los/as cultivadores/as.

\begin{tabular}{|c|c|c|c|}
\hline \multicolumn{2}{|c|}{ Variables de segmentación } & \multirow{2}{*}{$\frac{\mathbf{n}}{52}$} & \multirow{2}{*}{$\frac{\%}{60,5 \%}$} \\
\hline & Hombre & & \\
\hline $\mathrm{SUAV}$ & Mujer & 34 & $39,5 \%$ \\
\hline \multirow{3}{*}{ Grupo de edad } & 18 a 34 años & 6 & $7,0 \%$ \\
\hline & 35 a 54 años & 40 & $46,5 \%$ \\
\hline & 55 años o más & 40 & $46,5 \%$ \\
\hline \multirow{5}{*}{ Nivel de estudios } & Sin acabar estudios primarios & 2 & $2,3 \%$ \\
\hline & Graduado escolar/ EGB/ ESO & 31 & $36,0 \%$ \\
\hline & Bachillerato/ BUP/ COU & 10 & $11,6 \%$ \\
\hline & Formación profesional de grado medio o superior & 18 & $20,9 \%$ \\
\hline & Estudios universitarios o superiores & 25 & $29,1 \%$ \\
\hline \multirow{6}{*}{ Situación laboral actual } & Trabajador/a & 38 & $44,2 \%$ \\
\hline & Estudiante & 3 & $3,5 \%$ \\
\hline & Desempleado/a & 20 & $23,3 \%$ \\
\hline & Jubilado/a o pensionista & 20 & $23,3 \%$ \\
\hline & Realiza tareas del hogar & 5 & $5,8 \%$ \\
\hline & Otras situaciones & 0 & $0 \%$ \\
\hline \multirow{3}{*}{$\begin{array}{l}\text { Auto-valoración de la situación } \\
\text { económica del hogar }\end{array}$} & Baja & 24 & $27,9 \%$ \\
\hline & Intermedia & 41 & $47,7 \%$ \\
\hline & Alta & 21 & $24,4 \%$ \\
\hline
\end{tabular}

Fuente: elaboración propia.

Se puede concluir, en términos generales, que la práctica del cultivo ha de entenderse como "transversal" atendiendo a las diferentes variables sociodemográficas consideradas. Bien es cierto que la diferente titularidad de los huertos analizados ("municipal" -Culleredo-y "asociativa" -Feáns-) matiza esta inicial "heterogeneidad" de perfiles. En este sentido, si se pone en relación la tipología de la titularidad de los huertos con las variables de segmentación, mediante consecutivas tablas de contingencia, los valores chicuadrado obtenidos ilustran varias correspondencias estadísticamente significativas. En concreto, estas se localizan al cruzar las titularidades de los huertos y los niveles forma- 
tivos estimados de la población, su situación laboral y la auto-valoración de la situación económica actual de sus hogares (véase Tabla II) ${ }^{17}$.

Tabla II. Caracterización de los/as cultivadores/as según huerto comunitario.

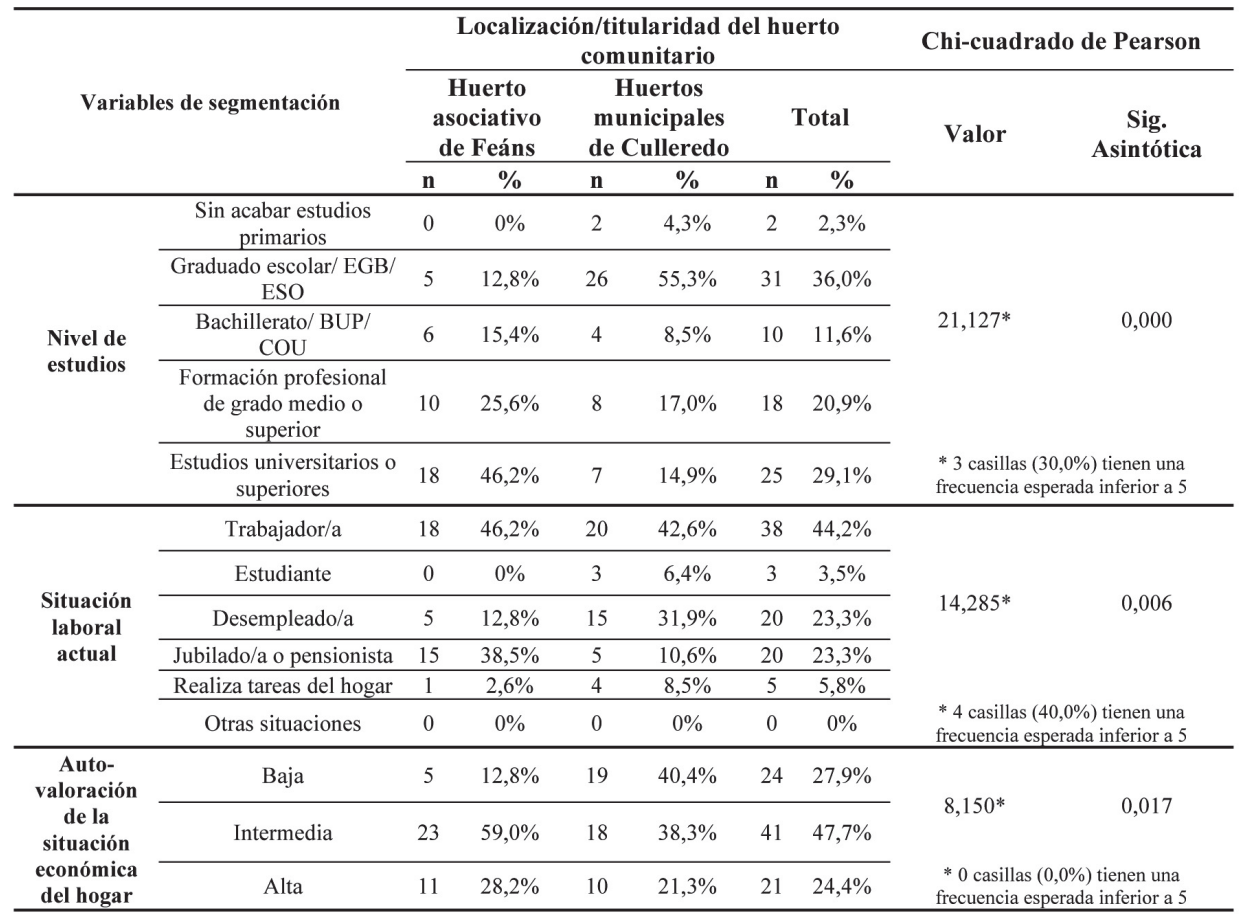

Fuente: elaboración propia.

Por tanto, en los huertos municipales de Culleredo prevalece un/a cultivador/a con un nivel formativo de primer o segundo grado $(55,3 \%)$, trabajador/a $(42,6 \%)$ o desempleado/a (31,9\%), con una auto-valoración de la situación económica de su hogar "baja" (40,4\%) o "intermedia" (38,3\%). Cabe precisar, como así puede ocurrir en otros casos, que la ordenanza reguladora de los mismos no observa una preferencia por ningún colectivo específico a la hora de conceder la titularidad de las parcelas (véase Tabla II).

Al contrario, en el huerto de Feáns, sobresale un perfil con un nivel de estudios universitario o superior $(46,2 \%)^{18}$, trabajador/a $(46,2 \%)$ o jubilado/a $(38,5 \%)$, con una valoración de su coyuntura económica "intermedia" $(59,0 \%)$ o "alta" (28,2\%) (véase Tabla II).

17 Se consideran significativos los valores de la prueba aparamétrica de bondad de ajuste $\mathrm{x}^{2^{*}}$ menores a 0,05 . En cualquier caso, el elevado porcentaje de frecuencias esperadas menores a 5, localizadas en dos de las tres tablas de contingencia presentadas, obliga a asumir con cautela estos resultados (véase Tabla II).

18 Este porcentaje de cultivadores/as con estudios universitarios es muy superior, atendiendo a los datos del censo de 2011, al del propio municipio de A Coruña $(23,4 \%)$ en el que está ubicado el huerto de Feáns. 
La diferencia en la titularidad de los terrenos, por tanto, puede ser un factor a tener en cuenta, y a estudiar con más detalle, para explicar la divergencia de perfiles detectados en la literatura española en HUPC de titularidad "no pública", con una probable mayor presencia de cultivadores/as pertenecientes a una "clase creativa" de elevado capital formativo y social (Simón, 2016; Michelini y Méndez, 2017); o "pública”, con usuarios/as que, seguramente, se ubican en coordenadas laborales y formativas diferentes (Ballesteros, 2016; Domene y Saurí, 2016).

Abordando la práctica cotidiana del cultivo, más de la mitad de las personas entrevistadas llevaban de uno a tres años yendo a su huerto en el momento de responder al cuestionario. La mayor parte acude durante todo el año, aunque de modo más intenso en la temporada de primavera y verano (el 75,6\% del total); solo un 5,8\% de los/as usuarios/ as interrumpen la actividad durante el otoño y el invierno. En este sentido, durante la época de más actividad la mayoría suele acudir varios días a la semana $(76,7 \%)$ y suele estar una media de 2 horas y 30 minutos en su parcela. Se observa por lo tanto que, aunque la asistencia a los huertos se concentra en el período primaveral y estival, la inversión de tiempo requerida es muy importante y ocupa un espacio temporal superior al de otras actividades sociales, deportivas, etc., en el contexto gallego (véase Tabla III) ${ }^{19}$.

Tabla III. Práctica cotidiana de la AUP en un huerto comunitario.

\begin{tabular}{|c|c|c|c|}
\hline \multicolumn{2}{|c|}{ Variables de práctica cotidiana } & \multirow{2}{*}{$\frac{\mathbf{n}}{13}$} & \multirow{2}{*}{$\frac{\%}{15,1 \%}$} \\
\hline \multirow{3}{*}{$\begin{array}{l}\text { Tiempo que se lleva acudiendo al } \\
\text { huerto }\end{array}$} & Un año o menos & & \\
\hline & De 1 a 3 años & 47 & $54,7 \%$ \\
\hline & Más de 3 años & 26 & $30,2 \%$ \\
\hline \multirow{4}{*}{$\begin{array}{l}\text { Frecuencia con la que se acude al } \\
\text { huerto en el transcurso del año }\end{array}$} & $\begin{array}{l}\text { Durante todo el año con, prácticamente, } \\
\text { la misma asiduidad }\end{array}$ & 14 & $16,3 \%$ \\
\hline & $\begin{array}{c}\text { Durante todo el año, aunque más } \\
\text { activamente en la temporada primavera } \\
\text { verano }\end{array}$ & 65 & $75,6 \%$ \\
\hline & $\begin{array}{c}\text { Durante el otoño invierno sólo de vez en } \\
\text { cuando }\end{array}$ & 2 & $2,3 \%$ \\
\hline & Sólo en la temporada primavera verano & 5 & $5,8 \%$ \\
\hline \multirow{3}{*}{$\begin{array}{l}\text { Frecuencia con la que se acude al } \\
\text { huerto en la temporada de mayor } \\
\text { actividad }\end{array}$} & Varios días a la semana & 66 & $76,7 \%$ \\
\hline & Uno o dos días a la semana & 18 & $20,9 \%$ \\
\hline & Algunos días al mes & 2 & $2,3 \%$ \\
\hline \multirow{5}{*}{$\begin{array}{c}\text { ¿Con quién sueles acudir a tu } \\
\text { huerto? } \\
\text { (respuesta múltiple) }\end{array}$} & Nadie & 59 & $68,6 \%$ \\
\hline & Cónyuge/pareja & 39 & $45,3 \%$ \\
\hline & Familiares & 22 & $25,6 \%$ \\
\hline & Amigos/as & 11 & $12,8 \%$ \\
\hline & Otros/as & 1 & $1,2 \%$ \\
\hline
\end{tabular}

Fuente: elaboración propia.

19 Por ejemplo, recogiendo datos de la última Encuesta de empleo del tiempo del INE (para el período 2009-2010), en Galicia se destina una media de 1 h 45 min a la vida social y diversión, 1 h 47 min a los deportes y actividades al aire libre, y $1 \mathrm{~h} 57 \mathrm{~min}$ a diferentes aficiones y actividades informáticas. 
Las personas suelen acudir en mayor medida con su pareja $(45,3 \%)$ o con familiares $(25,6 \%)$. Es relevante también constatar que un $12,8 \%$ de personas gestionan una huerta junto a amigos/as. A todos los efectos, se observa como la gestión de cada parcela es una práctica que suele ser llevada a cabo por más de una persona; no obstante, el porcentaje de personas que dicen acudir en ocasiones sin compañía es relevante $(68,6 \%)$ (véase Tabla III).

\subsection{Análisis de las motivaciones}

A continuación, se abordará la importancia que los/as cultivadores/as otorgan a las motivaciones que impulsan la práctica del cultivo periurbano. Estas se clasifican, abarcando la mayor parte de las líneas temáticas identificadas en la literatura, en tres grupos: "ociosas y relacionales", "pragmáticas" ${ }^{20}$ (derivadas del cultivo de alimentos y sus prácticas asociadas), y "simbólicas" o "ideológicas" (relacionadas, por ejemplo, con la defensa de valores medioambientales) ${ }^{21}$.

A través de 14 variables de escala, agrupadas nuevamente en estos tres bloques, se gradúa el nivel de acuerdo o no que gira alrededor de cada motivación (véase Gráfico 1).

En líneas generales, son las motivaciones "simbólicas" y "pragmáticas" las que suscitan un mayor grado de consenso a la hora de explicar la asistencia rutinaria a los huertos, alcanzando las puntuaciones medias más elevadas. Son las siguientes:

- La importancia genérica de apoyar este tipo de iniciativas (9,4 puntos de promedio).

- La calidad elevada de los alimentos cultivados $(9,0)$.

- El consumo de alimentos más sanos $(9,0)$.

- El desarrollo de una actividad respetuosa con el medio ambiente $(8,7)$.

Sin embargo, otros enunciados con medias inferiores (por ejemplo, el referido a la percepción del "ahorro de dinero" conseguido con los cultivos), presentan disensos importantes, tal y como ilustran las elevadas desviaciones típicas alcanzadas (véase Gráfico 1).

En consecuencia, es necesario analizar esta disparidad de medias a través de próximos análisis de varianza (en adelante ANOVA), que incluyan como factores tres grupos de edad (de 18 a 34 años, de 35 a 54 y 55 años o más) y las auto-valoraciones de la situación económica del hogar ("baja", "intermedia" y "alta”). Ambas variables son elegidas porque son las más reiteradas en la literatura citada a la hora de establecer los perfiles "tipo" de hortelanos/as que moldean la idiosincrasia actual de los HUPC.

20 Cabe precisar que la "dimensión pragmática" sigue siendo el eje principal de discusión sobre el que gira el discurso de la AUP en muchos puntos del planeta, y no se puede referir solo a países "en vías de desarrollo", sino también a lugares en países "desarrollados" donde existe una dificultad real de acceder, por precio y distancia, a hortalizas frescas (Morland et al., 2002).

21 Esta dicotomía de motivaciones ("pragmáticas" y "simbólicas") tiene su correspondencia teórica en las diferencias establecidas por Inglehart (1991) entre valores "materialistas" y "postmaterialistas". 
Gráfico 1. Valoraciones de las motivaciones (medias y desviaciones típicas -en blanco-).

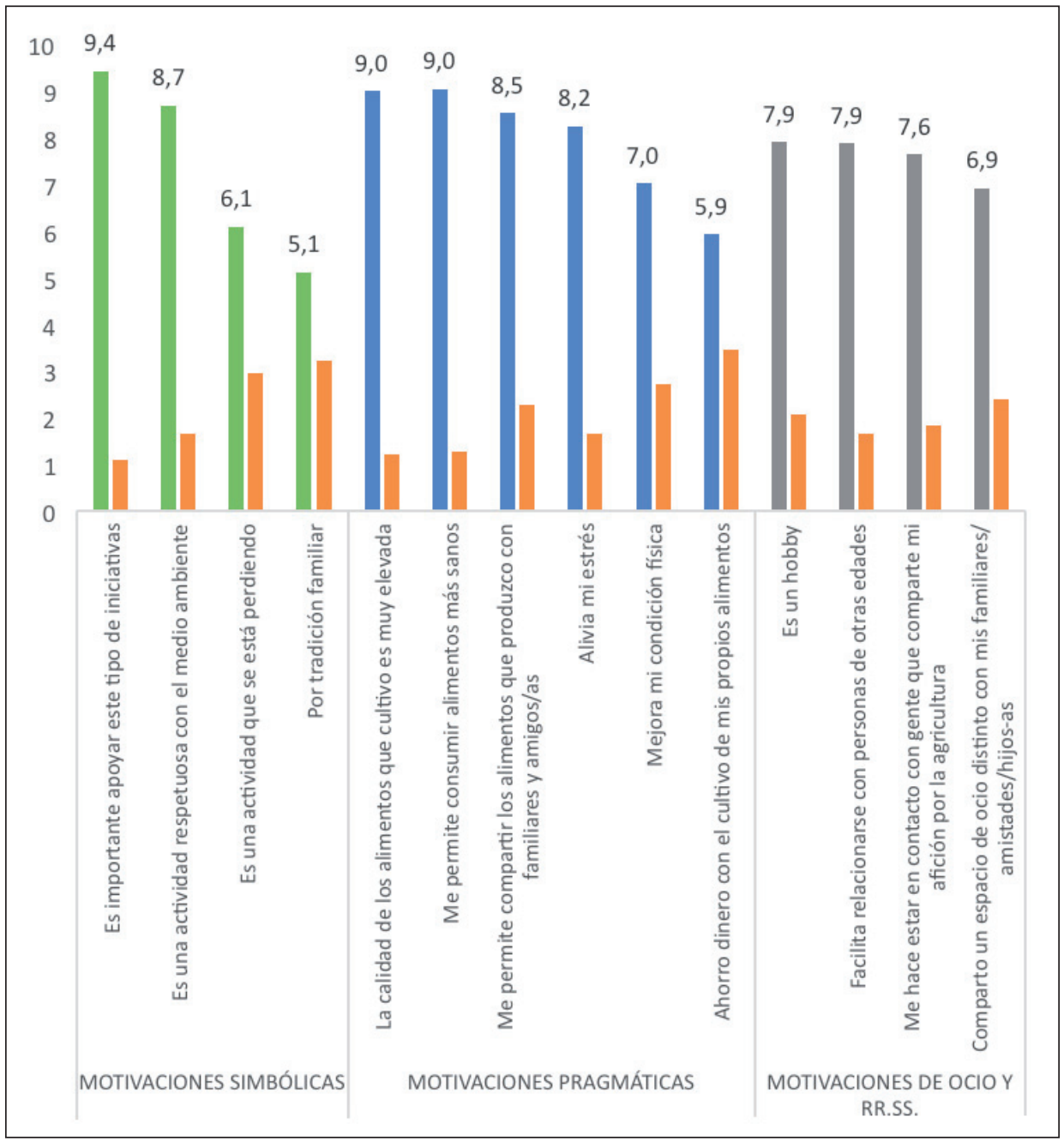

Nota: el valor 0 de la escala equivale a la expresión "totalmente en desacuerdo"; el valor 10 a la expresión "totalmente de acuerdo".

Fuente: elaboración propia.

Desagregando también el conjunto de estas puntuaciones atendiendo al carácter asociativo o municipal de los huertos, es importante señalar que, tras realizar el pertinente contraste, no se localizó ninguna diferencia de medias significativa ${ }^{22}$. Por tanto, la titulari-

22 De hecho, el valor de F más alto $(3,205)$ conseguido en el contraste del enunciado "la actividad alivia mi estrés", obtiene una significación igual a 0,077. Por tanto, no debe rechazarse el supuesto de igualdad de medias. 
dad no induce en esta ocasión una valoración diferenciada en las motivaciones que rodean a esta particular práctica agrícola. Esta casuística, a refrendar en próximas investigaciones, no coincide con las indagaciones de Veen et. al. (2015) acerca de la preeminencia del ocio y de las relaciones sociales en huertos gestionados de modo colectivo, con parcelas individuales no estrictamente delimitadas, y con terrenos de uso comunal -como sería el caso de Feáns-.

\subsubsection{Motivaciones y grupos de edad}

Mediante un ANOVA, se han identificado del total de ítems motivacionales (véase Gráfico 1), cinco en los que se establecen diferencias de medias significativas atendiendo a los grupos de edad de los/as usuarios/as de los huertos (véase Tabla IV para el conjunto de datos de este epígrafe).

Dentro de las motivaciones "simbólicas", las personas de mayor edad son las que más acuden al huerto para "evitar que la práctica agrícola se pierda". En este ítem, la diferencia de medias resulta significativa entre el grupo de 35 a 54 años y el de mayores de 55 años (5,2 puntos y 7,0 respectivamente).

Son también los/las de más edad quienes consideran importante mantener "la tradición familiar agrícola" a través del contacto con los huertos, estableciendo este colectivo una diferencia significativa en la valoración media de este ítem (6,5 puntos) respecto a la de los grupos de edad restantes (2,2 puntos y 4,2 respectivamente). La importancia de la agricultura como manifestación cultural ligada al pasado propio, solo cobra importancia en los/as cultivadores/as de más edad (que suponen el 46,5\% estimado del total). En conclusión, esta "meta" no es desde luego irrelevante, y ha sido también detectada en otros estudios (Domene y Saurí, 2016).

Tabla IV. Motivaciones percibidas por grupos de edad (contrastes de medias significativos).

\begin{tabular}{|c|c|c|c|c|c|c|c|c|c|c|}
\hline \multirow{2}{*}{\multicolumn{2}{|c|}{ MOTIVACIONES }} & \multicolumn{4}{|c|}{ Grupo de edad } & \multicolumn{2}{|c|}{ ANOVA } & \multicolumn{3}{|c|}{ Games-howell } \\
\hline & & $\begin{array}{c}18 \text { a } 34 \\
\text { años } \\
(1)\end{array}$ & $\begin{array}{c}35 \text { a } 54 \\
\text { años } \\
(2)\end{array}$ & $\begin{array}{l}55 \text { años } \\
\text { o más } \\
\text { (3) }\end{array}$ & Total & $\mathbf{F}$ & Sig. & $\begin{array}{l}\text { Relaciones } \\
\text { significativas }\end{array}$ & $\begin{array}{l}\text { Diferencia } \\
\text { de medias } \\
\text { (I-J) }\end{array}$ & Sig. \\
\hline \multirow{2}{*}{ SIMBÓLICA } & $\begin{array}{l}\text { Es una actividad que se } \\
\text { está perdiendo }\end{array}$ & 6,2 & 5,2 & 7,0 & 6,1 & 4,124 &, 020 & 2 y 3 & $-1,8250^{*}$ &, 016 \\
\hline & Por tradición familiar & 2,2 & 4,2 & 6,5 & 5,1 & 9,458 &, 000 & $\begin{array}{lll}1 & \text { y } 3 \\
2 \text { y } 3\end{array}$ & $\begin{array}{l}-4,3333^{*} \\
-2,3250^{*}\end{array}$ & $\begin{array}{l}, 003 \\
, 002\end{array}$ \\
\hline \multirow{2}{*}{ PRAGMÁTICA } & $\begin{array}{l}\text { Mejora mi condición } \\
\text { fisica }\end{array}$ & 2,5 & 5,9 & 8,8 & 7,0 & 37,493 &, 000 & $\begin{array}{l}1 \text { y } 2 \\
1 \text { y } 3 \\
2 \text { y } 3 \\
\end{array}$ & $\begin{array}{l}-3,4000^{*} \\
-6,3000^{*} \\
-2,9000^{*} \\
\end{array}$ & $\begin{array}{l}, 019 \\
, 001 \\
, 000 \\
\end{array}$ \\
\hline & $\begin{array}{l}\text { Ahorro dinero con el } \\
\text { cultivo de mis propios } \\
\text { alimentos }\end{array}$ & 3,0 & 5,1 & 7,2 & 5,9 & 6,687 &, 002 & 2 у 3 & $-2,0750^{*}$ & 013 \\
\hline $\begin{array}{l}\text { OCIO Y } \\
\text { RELACIONES } \\
\text { SOCIALES }\end{array}$ & $\begin{array}{c}\text { Comparto un espacio de } \\
\text { ocio distinto con mis } \\
\text { familiares / amistades / } \\
\text { hijos-as }\end{array}$ & 7,5 & 6,1 & 7,7 & 6,9 & 5,468 &, 006 & $\begin{array}{l}1 \text { y } 2 \\
2 \text { y } 3\end{array}$ & $\begin{array}{l}1,4500^{*} \\
-1,6500^{*}\end{array}$ & 011 \\
\hline
\end{tabular}


Al respecto de las motivaciones "pragmáticas", los contrastes más significativos se encuentran en la percepción del cultivo como una actividad que "mejora la condición física". Esta cuestión es más importante a medida que la edad aumenta, pasando de ser una de las razones menos valoradas para el grupo de 18 a 35 años (con una media de 2,5 puntos), a tener una importancia relativa en el grupo de 35 a 54 años $(5,9)$, para, finalmente, alcanzar una relevancia muy destacada en el grupo de edad de mayores de $55(8,8)$.

De igual modo, la importancia que tiene la actividad del cultivo en el "ahorro de dinero", aumenta a medida que lo hace la edad. La diferencia estadísticamente significativa se localiza en el grupo de 35 a 54 años (5,1 puntos) respecto al de los mayores de 55 años $(7,1)$.

Por último, el papel que el huerto tiene como espacio de ocio alternativo compartido con familiares, amigos/as e hijos/as, también presenta alguna particularidad. Son los grupos de edad extremos (18-34 años y 55 o más años), los que más valoran esta cualidad relacional que surge de la asistencia a los huertos.

\subsubsection{Motivaciones y situación económica percibida}

Nuevamente, gracias al rechazo significativo de la hipótesis de igualdad de medias mediante un ANOVA, podemos estudiar el comportamiento de la auto-valoración de la situación económica del hogar de cada cultivador/a, y determinados ítems motivacionales (véase Tabla V para el conjunto de datos de este epígrafe).

En cuanto a la dimensión "simbólica", las personas entrevistadas que gozan de una situación económica auto-percibida "alta" puntúan de modo superior, respecto de los dos colectivos con una situación "intermedia" o "baja", las motivaciones que subyacen al hecho de "apoyar iniciativas de este tipo" y de realizar una tarea "respetuosa con el medio ambiente"23. Una coyuntura inversa ocurre con el ítem que explica la asistencia a los huertos por mantener "una tradición familiar", aspecto muy relevante para colectivos de renta baja y de más edad -como se ha visto en párrafos anteriores-.

Sobre el ámbito "pragmático", se constata que a medida que desciende la valoración de la situación económica familiar, aumenta la consideración que se le da al "ahorro de dinero conseguido con el cultivo de mis propios alimentos". La relación es clara y las diferencias son significativas entre los tres grupos. Por ejemplo, las personas con una valoración de la situación económica del hogar "baja" consideran importante la motivación del "ahorro" (7,5 puntos), mientras que las personas con una valoración "alta" $(3,1)$ o "intermedia" $(6,4)$ le dan una importancia menor.

23 Respecto a la significación del contraste de medias, en estas dos variables cabe decir que no se cumple el supuesto de normalidad propio de un ANOVA. 
Tabla V. Motivaciones percibidas por auto-valoración de la situación económica del hogar (contrastes de medias significativos).

\begin{tabular}{|c|c|c|c|c|c|c|c|c|c|}
\hline \multirow{2}{*}{\multicolumn{2}{|c|}{ MOTIVACIONES }} & \multicolumn{3}{|c|}{$\begin{array}{l}\text { Auto-valoración de la } \\
\text { situación económica del } \\
\text { hogar }\end{array}$} & \multicolumn{2}{|c|}{ ANOVA } & \multicolumn{3}{|c|}{ Games-howell } \\
\hline & & $\begin{array}{c}\text { Baja } \\
(1)\end{array}$ & $\begin{array}{l}\text { Intermedia } \\
\quad(2)\end{array}$ & $\begin{array}{c}\text { Alta } \\
(3)\end{array}$ & $\mathbf{F}$ & Sig. & $\begin{array}{l}\text { Relaciones } \\
\text { significativas }\end{array}$ & $\begin{array}{c}\text { Diferencia } \\
\text { de medias } \\
\text { (I-J) }\end{array}$ & Sig. \\
\hline \multirow{3}{*}{ SIMBÓLICA } & $\begin{array}{l}\text { Es importante apoyar este } \\
\text { tipo de iniciativas }\end{array}$ & 8,7 & 9,7 & 9,8 & 8,099 &, 001 & $\begin{array}{l}1 \text { y } 2 \\
1 \text { y } 3\end{array}$ & $\begin{array}{c}-, 9746^{*} \\
-1,0060^{*}\end{array}$ & $\begin{array}{l}018 \\
, 022\end{array}$ \\
\hline & $\begin{array}{c}\text { Es una actividad } \\
\text { respetuosa con el medio } \\
\text { ambiente } \\
\end{array}$ & 8,1 & 8,7 & 9,4 & 3,698 &, 029 & 1 y 3 & $-1,2976^{*}$ &, 031 \\
\hline & Por tradición familiar & 6,2 & 5,3 & 3,6 & 4,005 &, 022 & 1 y 3 & $2,5952^{*}$ &, 037 \\
\hline \multirow{2}{*}{ PRAGMÁTICA } & \multirow{2}{*}{$\begin{array}{c}\text { Ahorro dinero con el } \\
\text { cultivo de mis propios } \\
\text { alimentos } \\
\end{array}$} & \multirow{2}{*}{7,5} & \multirow[b]{2}{*}{6,4} & \multirow[b]{2}{*}{3,1} & \multirow[b]{2}{*}{12,268} & \multirow{2}{*}{, 000} & 1 y 3 & $4,3571^{*}$ &, 000 \\
\hline & & & & & & & 2 y 3 & $3,2718^{*}$ &, 003 \\
\hline $\begin{array}{l}\text { OCIO Y } \\
\text { RELACIONES } \\
\text { SOCIALES }\end{array}$ & Es un hobby & 6,9 & 8,1 & 8,5 & 4,468 &, 014 & 1 y 3 & $-1,6488^{*}$ &, 025 \\
\hline
\end{tabular}

Fuente: elaboración propia.

Indagando en esta motivación económica, la mayoría de respuestas de las personas que dicen ahorrar algo en alimentación, se concentran en el intervalo de " 0 a $50 €$ durante el mes más productivo", con porcentajes también relevantes en el tramo de "50 a 100€" para los grupos de renta percibida "baja" e "intermedia" (véase Tabla VI). En definitiva, tal y como se desprende de la significación asociada al valor de chi-cuadrado ${ }^{24}$, existe una correspondencia entre la capacidad de ahorro y el nivel de renta percibida. A la espera de nuevas indagaciones en el contexto gallego, estos resultados no coinciden con la poca importancia atribuida al ahorro en el estudio de hortelanos/as jubilados/as realizado por Cabo et al. (2014: 75).

Tabla VI. Ahorro en alimentación (el mes que más produce el huerto) según auto-valoración de la situación económica del hogar.

\begin{tabular}{|c|c|c|c|c|c|c|c|c|c|c|}
\hline \multirow{3}{*}{$\begin{array}{c}\text { ¿Podrías decirme cuánto } \\
\text { puedes ahorrar al mes en } \\
\text { alimentación, } \\
\text { aproximadamente, el mes } \\
\text { que más produce tu } \\
\text { huerto? }\end{array}$} & \multicolumn{8}{|c|}{ Auto-valoración de la situación económica del hogar } & \multicolumn{2}{|c|}{ Chi-cuadrado de Pearson } \\
\hline & \multicolumn{2}{|c|}{ Baja } & \multicolumn{2}{|c|}{ Intermedia } & \multicolumn{2}{|c|}{ Alta } & \multicolumn{2}{|c|}{ Total } & \multirow[b]{2}{*}{ Valor } & \multirow[b]{2}{*}{ Sig. Asintótica } \\
\hline & $\mathbf{n}$ & $\%$ & $\mathbf{n}$ & $\%$ & $\mathbf{n}$ & $\%$ & $\mathbf{n}$ & $\%$ & & \\
\hline Menos de $50 €$ al mes & 15 & $62,5 \%$ & 18 & $43,9 \%$ & 6 & $28,6 \%$ & 39 & $45,3 \%$ & \multirow{4}{*}{$16,519^{*}$} & \multirow{4}{*}{0,030} \\
\hline Entre 50 y $100 €$ al mes & 6 & $25,0 \%$ & 8 & $19,5 \%$ & 2 & $9,5 \%$ & 16 & $18,6 \%$ & & \\
\hline Entre 100 y $200 €$ al mes & 0 & $0,0 \%$ & 2 & $4,9 \%$ & 0 & $0,0 \%$ & 2 & $2,3 \%$ & & \\
\hline Más de $200 €$ al mes & 0 & $0,0 \%$ & 0 & $0,0 \%$ & 1 & $4,8 \%$ & 1 & $1,2 \%$ & & \\
\hline 5,0 & 3 & $12,5 \%$ & 13 & $31,7 \%$ & 12 & $57,1 \%$ & 28 & $32,6 \%$ & $\begin{array}{l}* 8 \text { casilla } \\
\text { frecuencia }\end{array}$ & $\begin{array}{l}53,3 \%) \text { tienen una } \\
\text { sperada inferior a } 5\end{array}$ \\
\hline
\end{tabular}

Fuente: elaboración propia.

24 El cómputo de casillas con frecuencias esperadas inferiores a 5 es elevado (véase Tabla VI). Por tanto, estamos hablando de una correspondencia entre variables categóricas débil. 
Por último, cabe decir que no existe una relación significativa entre el "ahorro en el mes más productivo" y la localización de los huertos de Culleredo y Feáns. De nuevo, las diferentes titularidades de los espacios agrícolas estudiados no afectan a esta dimensión motivacional "pragmática".

\section{CONCLUSIONES}

A través de esta investigación se ha llevado a cabo una aproximación al estudio de la agricultura periurbana en tres huertos comunitarios localizados en los municipios de A Coruña y Culleredo. La casi total ausencia de estudios sobre HUPC en Galicia, ha obligado a presentar un repaso previo de contribuciones en el ámbito internacional y nacional, sobre las que contrastar las evidencias empíricas recabadas. También, ha resultado necesario aportar datos inéditos (y provisionales) sobre la distribución geográfica, y los principales tipos de titularidades, de los HUPC en el contexto territorial autonómico.

Partiendo de una investigación exploratoria apoyada en la realización y análisis de una encuesta, se han propuesto tres objetivos generales. El primero de ellos ha sido el de establecer un perfil de cultivador/a en los tres huertos comunitarios. En términos agregados, este se caracteriza por su "heterogeneidad" atendiendo a las diferentes variables de segmentación utilizadas. No obstante, se ha determinado que las diferentes titularidades de los huertos discriminan dos tipos de perfiles de usuarios/as: primero, el del huerto asociativo de Feáns, en el que predomina un colectivo con una condición económica "intermedia" o "alta", de elevado capital formativo; segundo, el de los huertos municipales de Culleredo, en que el que sobresalen los/as hortelanos/as de hogares con una situación económica "baja" o "intermedia", con un nivel formativo inferior, y en el que se concentra un importante volumen de desempleados/as.

Como segundo objetivo, se ha analizado un detallado abanico de motivaciones ("pragmáticas", "simbólicas", “ociosas o relacionales") que apoyan la rutina de acudir a los huertos. La primera conclusión relevante es que la práctica de estos huertos es claramente multifuncional (Taylor, 2010), esto es, las personas entrevistadas valoran positivamente todos los aspectos contemplados en cada una de las tres dimensiones motivacionales (por ejemplo, la importancia de apoyar iniciativas de AUP, el respeto por el medioambiente que lleva implícito esta práctica, la calidad de los alimentos que se obtienen de la misma, su utilidad para entablar relaciones sociales, etc.) (véase Gráfico 1). Sin embargo, atendiendo a las variables de edad y de situación económica auto-percibida (ítems de gran relevancia en los análisis reflejados en la literatura consultada), se pueden matizar dos aspectos importantes:

- La motivación "pragmática" de "mejorar la condición física" acudiendo a los huertos destaca con claridad en el colectivo de mayores de 55 años (estableciéndose una clara y significativa diferencia respecto de las consideraciones de los otros dos grupos de edad más jóvenes). 
- El "ahorro percibido en alimentos" que implica la producción del huerto, resulta importante para el colectivo de cultivadores con una auto-valoración "baja" de la situación económica del hogar, así como para los/as usuarios/as de mayor edad.

También, se ha podido comprobar como el tiempo que se invierte en esta actividad es importante, y ha de considerarse a todos los efectos una práctica que modifica y estructura de manera considerable la rutina de la mayoría de las personas que participan de ella.

En definitiva, el principal hallazgo de este estudio de caso, y a la espera de promover nuevos trabajos en los que desarrollar una sistematización más robusta de la clase social, responde a la constatación de que las motivaciones analizadas son importantes para todos los grupos analizados. No obstante, las de tipo "pragmático" cobran mayor relevancia en los/as hortelanos/as de más edad y de peor condición económica, mientras que otros aspectos "simbólicos" o "postmateriales" aumentan su importancia en personas con un perfil sociodemográfico casi opuesto. Esta cuestión no es menor; de hecho, esta interacción en sí misma apuntala un argumento poderoso para seguir consolidando y aumentando las iniciativas de AUP, especialmente de naturaleza "municipalista”, en toda Galicia. 


\section{BIBLIOGRAFÍA}

Alaimo, K. et al. (2008). Fruit and vegetable intake among urban community gardeners. Journal of Nutrition Education and Behavior, 40(2), 94-101.

Ballesteros, G. (2016). Agricultura urbana en España: situación actual y perspectivas (Presentación). Jornada sobre agricultura urbana para una ciudad sostenible. Madrid: Observatorio de Agricultura Urbana. Recuperado de: http://www.itd.upm. es/resumen-y-presentaciones-de-la-jornada-agricultura-urbana-para-una-ciudadsostenible/.

Bisquert i Pérez, Kylyan Marc (2013). As Hortas Municipais de Belvís (Santiago de Compostela): unha experiencia de Educación Ambiental? Estudo do caso (Trabajo de Fin de Grado). Santiago de Compostela, Repositorio Minerva: Universidade de Santiago de Compostela.

Bisquert i Pérez, Kylyan Marc (2017). A Rede de Hortas Municipais de Santiago de Compostela (Galiza) e as súas implicacións para a Educación Ambiental. Ambientalmente sustentable. Revista científica galego-lusófona de educación ambiental, I(23-24), 141-163.

Cabo, V. et al. (2014). Análisis de las motivaciones para cultivar un huerto urbano: el caso de los jubilados de Valladolid (España). Revista Española de Estudios Agrosociales y Pesqueros, 239, 57-85.

Camps-Calvet, M. et al. (2015). Sowing resilience and contestation in times of crises: The case of urban gardening movements in Barcelona. Partecipazione e Conflitto, $8(2), 417-442$.

Clinton, N. et al. (2018). A global geospatial ecosystem services estimate of urban agriculture. Earth's Future, 6, 40-60, https://doi.org/10.1002/2017EF000536.

Domene, E. y Saurí, D. (2016). Urbanization and class-produced natures: Vegetable gardens in the Barcelona Metropolitan Region. Geoforum, 38, 287-298.

Duží, B. et al. (2017). The geography of urban agriculture: New trends and challenges. Moravian Geographical Reports, 25(2), 130-138.

Glover, T. et al. (2005). Association, sociability, and civic culture: The democratic effect of community gardening. Leisure Sciences, 27(1), 75-92.

Guitart, D. et al. (2012). Past results and future directions in urban community gardens research. Urban Forestry \& Urban Greening, 11, 364-373.

Inglehart, R. (1991). El cambio cultural en las sociedades industriales avanzadas. Madrid: Siglo XXI.

Integrated Food Security Support Service (2011). The place of urban and peri-urban agriculture (UPA) in national food security programmes. Rome: FAO.

McEldowney, J. (2017). Urban agriculture in Europe. Patterns, challenges and policies. Bruselas: European Parliamentary Research Service.

Michelini, J.J. y Méndez, R. (2017). Movilización social y alternativas alimentarias en áreas urbanas: los grupos de consumo agroecológico en Madrid. Ciudad y territorio. Estudios territoriales, 194, 679-698. 
Morán, N. y Aja, A. (2011). Historia de los huertos urbanos. De los huertos para pobres a los programas de agricultura urbana ecológica. I Congreso Estatal de Agricultura Ecológica Urbana y Periurbana. Elche: SEAE. Recuperado de: http://oa.upm. es/12201/1/INVE_MEM_2011_96634.pdf.

Morán, N. (2009). Huertos urbanos en tres ciudades europeas: Londres, Berlín, Madrid. Boletín $C F+S$, 47/48, 75-124. Recuperado de: http://habitat.aq.upm.es/boletin/ n47/anmor_1.html.

Morland, K. et al. (2002). Neighborhood characteristics associated with the location of food stores and food service places. American Journal of Preventive Medicine, 22(1), 23-29.

Mougeot, L.J.A. (2005). Introduction. En Mougeot, L.J.A. (ed.), Agropolis: The social, political, and environmental dimensions of urban agriculture (pp. 1-24). London: Earthscan.

Mougeot, L.J.A. (2011). International support to research and policy on urban agriculture (1996-2010): Achievements and challenges. Urban Agriculture Magazine, 25, 12-17.

Pudup, M., B. (2008). It takes a garden: Cultivating citizen-subjects in organized garden projects. Geoforum, 39, 1228-1240.

Puente, R. (2012). Los huertos urbanos de Sevilla: de la tradición a la novedad. Sevilla: Diputación de Sevilla.

Simón, M. (2016). El consumo desde lo colectivo. Lectura en clave de sostenibilidad. Soberanía alimentaria, biodiversidad y culturas, 27, 35-38.

Simón, M. (2017). La alimentación como vector de intervención sociourbanística: agroecología y tejido productivo de barrio. En Equipo Red de Saberes (eds.), Hacia un Plan Integral de Barrio. Universidad Popular de Bellas Vistas (pp. 79-88). Madrid: Distrito de Tetuán. Ayuntamiento de Madrid.

Soriano, J. (2017). Motivaciones, usos y beneficios de la agricultura urbana: un estudio de caso en tres huertos comunitarios del área perimetropolitana de A Coruña (Trabajo de fin de máster). A Coruña: Universidade da Coruña.

Taylor, S. (2010). Multifunctional urban agriculture for sustainable land use planning in the United States. Sustainability, 2, 2499-2522.

Veen, E.J. et al. (2015). Community gardening and social cohesion: Different designs, different motivations. Local Environment, 21(10), 1271-1287.

Verdaguer, C. (2013). El campo y la ciudad, áreas de reencuentro. Hacia una Nueva Cultura del Territorio. Habitat y Sociedad, 6, 11-40. 